\title{
Hemolytic Uremic syndrome associated with pregnancy: Outcome from acute Kidney Injury
}

\author{
Rubina Naqvi
}

\begin{abstract}
Objective: To report here, case series of women developing acute kidney injury (AKI) in association with hemolytic uremic syndrome (HUS) during pregnancy or within days postpartum.

Methods: Subjects for the study reported here comprised of a cohort of 49 women referred from OBGYN and diagnosed having AKI and HUS. AKI was defined according to RIFLE criteria and HUS on basis of hematological, biochemical and histological features. All patients had normal size kidneys on ultrasonography and no previous co- morbidity.

Results: From January 2000 - January 2020, 49 such women were admitted/ referred to this facility. The mean age of these patients was $29.02 \pm 5.258$ years. Two had HUS during pregnancy while rest during postpartum. Majority of women had more than one insults these include hemorrhages, intrauterine deaths, operative measure (lower segment caesarean section). Renal replacement was required in all women. Complete renal recovery was observed in 14 patients, while one died during acute phase of illness. CKD-V developed in 17 patients, 16 patients lost long term follow up, but were dialysis free till last follow up and one left against medical advice during acute phase of illness. Treatment with plasmapheresis revealed significantly better renal recovery ( $p$ value 0.03 ) in this group of patients.

Conclusion: AKI with background of Hemolytic Uremic Syndrome (HUS) may remain irreversible in many of these young women. Plasmapheresis should be offered to patients with established diagnosis of HUS.
\end{abstract}

KEYWORDS: AKI, RIFLE criteria, Hemolytic Uremic Syndrome, Pregnancy, Plasmapheresis.

doi: https://doi.org/10.12669/pjms.36.6.2931

How to cite this:

Naqvi R. Hemolytic Uremic syndrome associated with pregnancy: Outcome from acute Kidney Injury. Pak J Med Sci. 2020;36(6):1153-1157. doi: https://doi.org/10.12669/pjms.36.6.2931

This is an Open Access article distributed under the terms of the Creative Commons Attribution License (http://creativecommons.org/licenses/by/3.0), which permits unrestricted use, distribution, and reproduction in any medium, provided the original work is properly cited.

\section{INTRODUCTION}

1. Prof. Dr. Rubina Naqvi

Department of Nephrology,

Sindh Institute of Urology and Transplantation,

Karachi, Pakistan.

Correspondence:

Prof. Dr. Rubina Naqvi

Department of Nephrology,

Sindh Institute of Urology and Transplantation (SIUT),

Karachi. 74200. Pakistan.

E-mail: naqvirubina@yahoo.com rubinanaqvi@gmail.com

* Received for Publication:

May 20, 2020

* Revision Received:

* Revision Accepted:

August 5, 2020

Pak J Med Sci September - October 2020

Early theories in pathogenesis of HUS proposed occlusion of arterioles with fibrin, causing mechanical destruction of platelets and red blood cells while passing through these occluded arterioles. Abnormal plasma factors have also been postulated as the cause of intra vascular platelet activation in HUS. ${ }^{1}$ Further down, atypical hemolytic uremic syndrome during pregnancy or soon after child birth (PaHUS) is reported to be often associated with coagulation abnormalities with disseminated intra vascular coagulation in $30 \%$ of cases. Verotoxin producing E. Coli infection was also supposed to be one of possibility 
triggering PaHUS. ${ }^{2}$ With further advancement in research it was stated that uncontrolled complement activation during pregnancy can lead to thrombotic microangiopathy and PaHUS. ${ }^{3}$

The incidence of PaHUS has been reported 1 in 25,000 pregnancies and accounts for $20 \%$ of all HUS cases in women. ${ }^{4,5}$ We have previously reported $30.47 \%$ of total HUS was associated with pregnancy. ${ }^{6}$ Another study from country reported Pregnancy related AKI found HUS in $6.3 \%$ women. ${ }^{7}$

Risks of different forms of thrombotic microangiopathies including ADAMTS 13 deficiency associated HUS increase during pregnancy. ${ }^{8}$ During normal pregnancy complement activation occurs at site of placental attachment to uterine wall and alternate pathway regulation depends on CD 59 and Decay accelerating factor. ${ }^{9}$ PaHUS is a devastating disease where clinical understanding and treatment options are still limited..$^{10}$ It may develop as a complication of preeclamsia ${ }^{11}$ in association with HELLP syndrome or sometimes with extensive hemorrhage pre or post partum. ${ }^{12}$ Progressing to life threatening variant of preeclampsia with severe microangiopathic hemolytic anemia and thrombocytopenia along with AKI and liver damage in some women. In this situation coagulation may be found to be abnormal with disseminated intravascular coagulation. ${ }^{13}$ We aim to report our experience of PaHUS from a tertiary renal care center by giving their presentation, management and outcome.

\section{METHODS}

In this retrospective study we reviewed clinical records of all women who were brought to Sindh Institute of Urology and Transplantation (SIUT) from January 2000 - January 2020 and in whom the diagnosis of AKI and HUS during pregnancy or in post partum period was established.

In this study AKI was defined according to RIFLE criteria; and all patients fall in one of category between ' $R$ ' and ' $L$ ', though patients referred here in ' $R$ ' category are very few and were mainly from OBGYN department of same hospital (Civil Hospital, Karachi).

As previously described; 6 HUS was defined as association of following criteria: mechanical hemolytic anemia (hemoglobin $<11 \mathrm{~g} / \mathrm{dl}$, raised lactate dehydrogenase, presence of schistocytes or fragmented red blood cells on blood peripheral film) and thrombocytopenia (platelet count $<150 \times 10^{3} / \mu \mathrm{l}$ ). All patients had renal biopsy done and diagnosis of HUS was confirmed on biopsy when met the criteria of fragmented RBC, extravasations of red blood cells, obliteration of capillary lumina and presence of fibrin thrombi. (Fig.1) Patient demographics were recorded for laboratory parameters of CBC, renal chemistry, liver function tests, coagulation profile, urinalysis, serum LDH and complement levels. Factor H; which is done by ELISA method in our laboratory was available for patients who were registered after March 2015. Management of all patients was recorded for need for renal replacement therapy (RRT), plasmapheresis (PP), its duration and finally outcome. Only data from patients fulfilling criteria of definitions for AKI and HUS, be it hematological/ biochemical or histological, were included in the study.

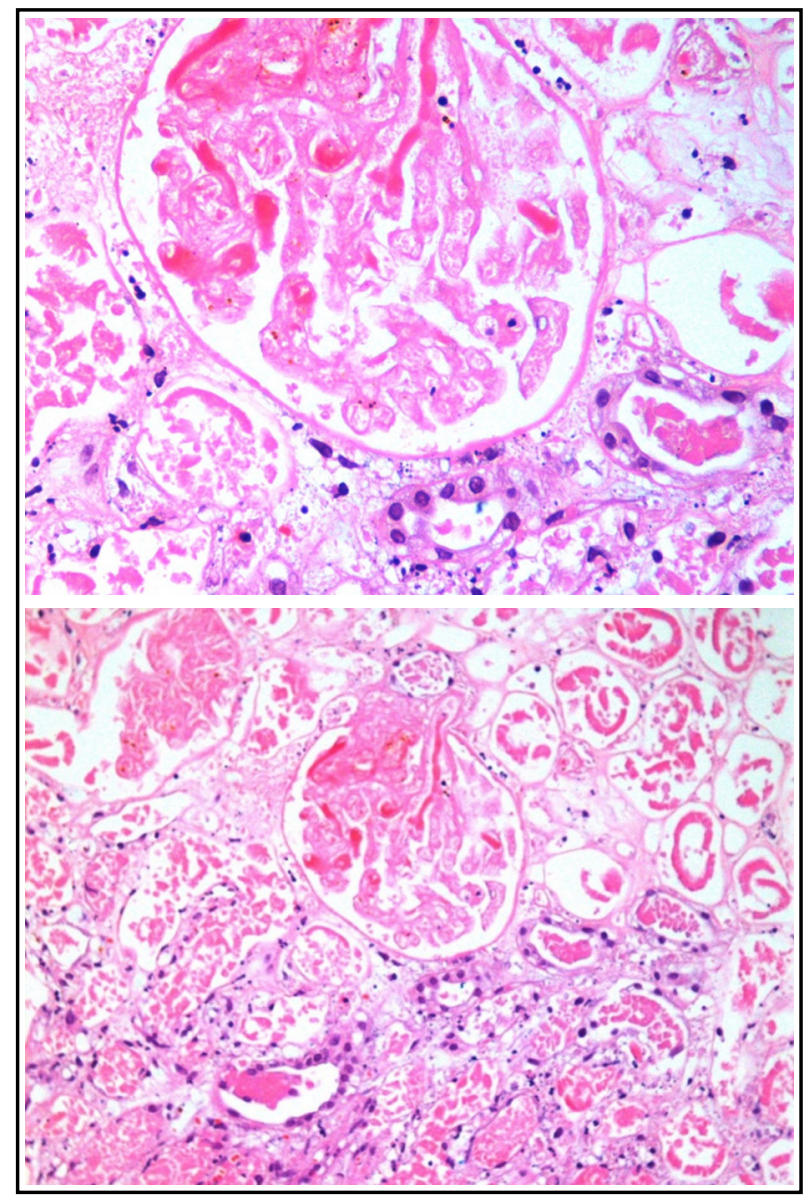

Fig.1: Histological findings in two different magnifications.

High-power view showing an infarcted glomerulus with dark red colored fibrin thrombi in some glomerular capillaries. A few tubules are still viable in the lower part of the field. (H\&E, ×400).

Medium-power view showing an infarcted glomerulus with fibrin thrombi in some glomerular capillaries. A few tubules are still viable in the lower part of the field. (H\&E, $\times 200)$. 
All patients were followed up till death, complete renal recovery or no recovery (labeled as $\mathrm{CKD}-\mathrm{V})$. Patients with partial recovery of kidney function and who lost to follow up were considered among the partial recovery of patients. Patients with HELLP syndrome or other identifiable cause of secondary HUS, like lupus or antiphospholipid syndrome were excluded from study.

Informed consent for starting RRT, doing biopsy, proceeding with PP or sharing information without personal identification was achieved from all women or their designated family member. Verbal consent from ERC of institution was taken as data was retrieved from records documented process was not suggested by ERC.

Statistical Methods: Statistical analysis was done on SPSS version 20.0 (IBM, USA). Mean \pm standard deviation was expressed or median with inter-quartile range provided for continuous variables. Frequencies and percentages computed for categorical variables. The chi square test of independence was used to determine the proportional difference between outcomes with different presenting symptoms or outcome with provision of plasmapheresis. P-value $<0.05$ was considered as significant.

\section{RESULTS}

During the studied period from January 2000 - January 2020, total 49 women were diagnosed to have AKI with Pa HUS. Basic demographics of studied population and initial laboratory parameters are given in Table-I.

Majority had more than one insult as contributing factors for example ante-partum hemorrhage (APH) as single factor was only found in one patient while in association with intra uterine death (IUD) of fetus in seven, APH, IUD with childbirth through lower segment caesarean section (LSCS) in 11, APH, IUD with postpartum hemorrhage (PPH) in three, $\mathrm{APH}$, IUD and PPH in three, APH with LSCS in two, IUD and LSCS in 2, IUD and PPH in two, LSCS with twin pregnancy in one patient and only LSCS in 5 women. Two patients developed hemeparesis after child birth and during course of illness in hospital. Two of 49 developed HUS during pregnancy while rest was post partum. Time from first day of symptom to reaching to our hospital was recorded as days of insult and given in Table-I.

Only one patient had non oliguric AKI, while 16 $(32.7 \%)$ had oliguria and $32(65.3 \%)$ were anuric on presentation. Renal replacement therapy (which was done in form of hemodialysis) was required in all patients.

The urinalysis during hospital stay was possible in $37(75.5 \%)$ women, rest had absolute anuria. Among these all had microscopic hematuria and $1-4+$ protein on dipstick. Deranged International Normalized Ratio (INR) was found in only one patient at time of admission. Renal biopsy was performed in 48 of 49 patients, in 21 biopsies along with fragmentation and extravasation of red blood

Table-I: Baseline demographics and laboratory values.

\begin{tabular}{lccc}
\hline Parameters & Mean $\pm S D$ & Median & Inter Quartile Range \\
\hline Age (years) & $29.02 \pm 5.258$ & 29 & $25-33$ \\
Duration of Insult (days) & $8.39 \pm 9.673$ & 6 & $2.5-10$ \\
Hemoglobin $(11.5-15.4 \mathrm{G} / \mathrm{dl})$ & $7.516 \pm 1.710$ & 6.2 & $6.2-8.9$ \\
Total leukocyte count $\left(4-11 \times 10^{3} / \mu \mathrm{l}\right)$ & $19.58 \pm 9.252$ & 9.5 & $12.55-24.50$ \\
Platelet $\left(150-400 \times 10^{3} / \mu \mathrm{l}\right)$ & $152.08 \pm 122.62$ & 70 & $70-209.50$ \\
Urea $(15-39 \mathrm{mg} / \mathrm{dl})$ & $166.96 \pm 75.219$ & 119 & $117-209$ \\
Creatinine $(\mathrm{mg} / \mathrm{dl})(0.5-1.5)$ & $8.734 \pm 4.027$ & 7.790 & $5.7-10.8$ \\
Sodium $(136-156 \mathrm{meq} / \mathrm{l})$ & $134.71 \pm 7.789$ & 136 & $132-138$ \\
Potassium $(3.5-5.1 \mathrm{meq} / \mathrm{l})$ & $4.837 \pm 0.977$ & 4.6 & $4.15-5.35$ \\
LDH $(<247 \mathrm{U} / \mathrm{L})$ & $2421.92 \pm 1190.239$ & 2208 & $1435-3273$ \\
Total Bilirubin $(0.2-1.0 \mathrm{mg} / \mathrm{dl})$ & $2.06 \pm 3.622$ & 0.7 & $0.56-1.43$ \\
AST $(10-42 \mathrm{U} / \mathrm{L})$ & $145.61 \pm 226.296$ & 55 & $29-180$ \\
ALT $(10-40 \mathrm{U} / \mathrm{L})$ & $105.49 \pm 144.760$ & 49 & $20-141.50$ \\
Factor H(250-600 mcg/ml) & $425.93 \pm 333.188$ & 291 & $111.75-762.25$ \\
C3 $(0.79-1.52 \mathrm{~g} / \mathrm{L})$ & $1.051 \pm 0.990$ & 0.99 & $0.805-1.235$ \\
\hline
\end{tabular}

$\mathrm{LDH}=$ Lactate dehydrogenase, $\mathrm{AST}=$ Aspartate Aminotransferase,

$\mathrm{ALT}=$ Alanine Aminotransferase, $\mathrm{C} 3=$ complement factor. 
Table-II: Predictors for Outcome.

\begin{tabular}{lc}
\hline Predictor & P value \\
\hline Oliguria & 0.605 \\
Anuria & 0.163 \\
Days of Insult & 0.939 \\
Mean of Platelet count on presentation & 0.739 \\
Mean of Urea on presentation & 0.744 \\
Mean of serum creatinine on presentation & 0.752 \\
Plasmapheresis done & 0.03 \\
\hline
\end{tabular}

${ }^{*}$ chi sq test of independence.

cells, obliteration of capillary lumina and presence of fibrin thrombi it revealed focal cortical necrosis, diffuse cortical necrosis in one and acute tubular necrosis in three. One biopsy along with fibrin thrombi revealed some chronic sclerosing changes in glomeruli.

One of $49(2 \%)$ patient died during acute phase of illness, one left against medical advice when explained about plasmapheresis and expected outcome, $14(28.6 \%)$ got complete renal recovery, $17(34.7 \%)$ remained dialysis dependent beyond 90 days and 16 (32.7\%) were patients who were dialysis free but not achieved normal renal functions yet and lost long term follow-up. Among those who recovered 6- 13 hemodialysis sessions were required. Plasmapheresis (PP) was not offered to 12 patients of these two were the women registered during very initial part of study, some refused for the therapy by family and for some reason was not clear from records. Later an average of 14 sessions of PP done in all and 22 sessions in one who developed HUS during pregnancy, though she recovered normal renal functions after initial phase of therapy but PP continued fortnightly till four weeks postpartum.

While other factors remained statistically insignificant to renal outcome it was found that provision of PP was found statistically significant and useful in renal recovery. (Table-II)

\section{DISCUSSION}

This is the largest to date study from our country assessing the presentation, management and outcome in PaHUS. Previously we have published a series of AKI secondary to HUS which included all causes; we have not performed complement genetic analysis on these patients so cannot comment on the fact whether PaHUS and other causes HUS differ in this regard. ${ }^{6}$ But others have reported no significant association of complement genetic make with PaHUS. ${ }^{10}$ The targeted inhibition of the complement alternative pathway has been worked out and some promising results have been described in some studies., ${ }^{4,12,14}$ The use of anti C5 monoclonal antibody, eculizumab has been published in reflecting its results in both PaHUS and other causes HUS, some have shown successful treatment with use of eculizumab with normalization of all hematologic features and rapid renal recovery. ${ }^{10}$ But main hindrance in use of this monoclonal antibody is its cost and non availability in many countries like ours. Many studies done before trials of eculizumab have shown PP as effective treatment in removing overactive proteins and functionally defective proteins. ${ }^{15-17}$

Plasma exchange has been suggested first line therapy for typical HUS associated with severe renal insufficiency or brain impairment for last many years and early plasmapheresis, with or without eculizumab, was supposed to remain the cornerstone of treatment. ${ }^{18}$ We have seen significantly good prognosis in terms of renal recovery in all causes of HUS and in present PaHUS. ${ }^{6}$ An interesting finding in our experience of AKI secondary to HUS was that during initial years of study we mostly use to see HUS with other causes and except for two rest of cases in present study were brought here during last four years, reason for this cannot be explained on scientific grounds. All histological samples were analyzed at our own histopathology department by same team members thus question of this variation is waived here.

Previously a Spanish study has shown an association of LSCS with PaHUS, ${ }^{10}$ in present series 22 of $49(45 \%)$ women has had LSCS done, in 5 of these 22 LSCS was only insult while in others it was associated with one or more than one other factor described above in results.

In present study which is analysis of retrospective data, some women were not considered for plasmapheresis (especially during early part of study), reason for which is not clear in records but it was evident that those who were offered this therapy revealed statistically significant better renal outcome ( $p$ value 0.03 ).

In a study in 2014 from Rawalpindi, Pakistan, among pregnancy associated AKI 13/88(15\%) had HUS, however their outcome was not mentioned. ${ }^{19}$ In present study we have seen complete renal recovery in $28.6 \%$ and irreversible renal failure in $34.7 \%$ women.

From same institution we have previously published large series of pregnancy associated AKI 
where over a span of 25 years 1,441 such women were treated but there HUS and HELLP were described under one category among many others. ${ }^{20}$ Another study from country reported 111 patients with pregnancy associated AKI, of these seven were diagnosed with HUS (3 had concomitant preeclampsia). However, no further data related to morbidity and mortality was mentioned. ${ }^{7}$

Limitations of study: Genetic screening and testing for autoantibodies were not performed in these patients. Monoclonal antibody Eculizumab was not used in any of these patients as it is not available in country.

\section{CONCLUSION}

Despite limitations with current study it is evident from data that prognosis in terms of renal recovery is not very good in AKI and HUS in obstetrical scenario where all patients are young. It is important to know the burden of disease so that future diagnostics and treatment modalities can be planned. PP should be offered to all women developing PaHUS especially when Eculizumab is not available or affordable.

Acknowledgement: Valuable help of Prof. Muhammad Mubarak (JIK Histopathology Lab. SIUT) for provision of histopathology photographs and their description and Mr. Iqbal Mujtaba (Research Dept. SIUT) for statistical analysis of data is gratefully acknowledged.

\section{Conflict of Interest: None.}

\section{REFERENCES}

1. Walters MDS, Levin M, Smith C, Nokes TJC, Hardisty RM, Dillon MJ, et al. Intrvascular platelet activation in the hemolytic uremic syndrome. Kidney Int. 1988;33(1):107115. doi: $10.1038 /$ ki.1988.17

2. Remuzzi G, Ruggenenti P. The hemolytic uremic syndrome. Kidney Int. 1995;47:2-19.

3. Noris M, Caprioli J, Bresin E, Mossali C, Pianetti G, Gamba $\mathrm{S}$, et al. Relative role of genetic complement abnormalities in sporadic and familial aHUS and their impact on clinical phenotype. Clin J Am Soc Nephrol. 2010;5(10): 1844-1859. doi: 10.2215/CJN.02210310

4. Noris M, Remuzi G. Atypical Hemolytic Uremic Syndrome. N Engl J Med. 2009;361:1676-1687. doi: 10.1056/ NEJMra0902814

5. Fakhouri F, Roumenina L, Provot F, Sallee M, Caillard S, Lionel Couzi L, et al. Pregnancy associated Hemolytic Uremic Syndrome revisited in era of Complement Gene Mutations. J Am Soc Nephrol. 2010;21(5):859-867. doi: 10.1681/ASN.2009070706
6. Naqvi R, Abbas K, Akhtar SF. Acute Kidney Injury with Hemolytic Uremic Syndrome: A Single Center Experience. Pak J Kidney Dis. 2020;11:193-198.

7. Haroon F, Murtaza F. Dhrolia, Qureshi R, Imtiaz S, Ahmed A. Frequency of Pregnancy-Related Complications Causing Acute Kidney Injury in Pregnant Patients at a Tertiary Care Hospital. Saudi J Kidney Dis Transpl. 2019;30(1):194-201.

8. Bruel A, Kavanagh D, Norris M, Delmas Y, Wong EKS, Bresin E, et al. Hemolytic Uremic Syndrome in Pregnancy and Postpartum. Clin J Am Soc Nephrol. 2017;12(8):12371247. doi: $10.2215 /$ CJN.00280117

9. Holmes $\mathrm{CH}$, Simps KL, Okada H, Wainright SD, Purcell DF, Houlihan JM. Complement regulatory proteins at the fetomaternat interface during human placental development: distribution of CD 59 by comparison with membrane co-factor protein (CD46) and decay accelerating factor (Cd55). Eur J Immunol. 1992;22(6):1579-1585. doi: 10.1002/ eji.1830220635

10. Huerta A, Arjona E, Portoles J, Lopez-Sanchez P, Rabasco C, Espinosa M, et al. A retrospective study of pregnancyassociated atypical hemolytic uremic syndrome. Kidney Int. 2018;93(2):450-459. doi: 10.1016/j.kint.2017.06.022

11. Lindheimer MD, Katz AI. Preeclampsia: Pathophysiology, diagnosis and management. Annu Rev Med.1989;40:233250. doi: 10.1146/annurev.me.40.020189.001313

12. Zuber J, Fakhouri F, Roumenina LT, Loirat C, Fre'meauxBacchi V, French Study Group for aHUS/C3G. Use of eculizumab for atypical haemolytic uraemic syndrome and C3glomerulopathies. Nat Rev Nephrol. 2012;8(11):643-657. doi: 10.1038/nrneph.2012.214

13. Gaber LW, Spargo BH, Lindheimer MD. Renal pathology in pre eclampsia. Clin Obstet Gynecol. 1987;1:971-975.

14. Dragon-Durey MA, Blanc C, Garnier A, Hofer J, Sethi SK, Zimmerhackl LB. Anti-factor H autoantibody-associated hemolytic uremic syndrome: review of literature of the autoimmune form of HUS. Semin Thromb Hemost. 2010;36(6):633-640. doi: 10.1055/s-0030-1262885

15. Ariceta G, Besbas N, Johnson S, Karpman D, Landau D, Licht $C$, et al. Guideline for the investigation and initial therapy of diarrhea negative hemolytic uremic syndrome. Pediat Nephrol. 2009;24(4):687-696. doi: 10.1007/s00467008-0964-1

16. Taylor CM, Machin S, Wigmore SJ, Goodship TH. Clinical practice guidelines for the management of atypical haemolytic uraemic syndrome in the United Kingdom. Br J Haematol. 2010;148(1):37-47. doi: 10.1111/j.13652141.2009.07916.x

17. Kavanagh D, Raman S, Sheerin NS. Management of hemolytic uremic syndrome. F1000Prime Rep. 2014;6:119. doi: 10.12703/P6-119

18. Ruggenenti P, Remuzzi G. A German outbreak of haemolytic uraemic syndrome. Lancet. 2011;378:1057-1058.

19. Khattak MI, Sabir S, Khattak SN, Tahir N. Spectrum of Acute Kidney Injury (AKI) in Pregnancy. J Rawal Med Coll. 2014;18(2):267-269.

20. Naqvi R, Ahmed E, Sheikh R, Rizvi A. Obstetrical Acute Kidney Injury: 25years experience from Nephrology Care Unit in Pakistan. Open Access Library J. 2015;2:e1778. doi: 10.4236/oalib.1101778 